\title{
Patellar Osteomyelitis in A 7-Year-Old Boy: A Case Report
}

\section{Eva DE Wachter*, Hisco Robijn, Pascal Wernaers and Nicole Gielissen}

Vrije Universiteit Brussel, Belgium

*Corresponding Author: Eva DE Wachter, Vrije Universiteit Brussel, Belgium.

Received: November 18, 2019; Published: November 26, 2019

DOI: $10.31080 /$ ASOR.2019.02.0124

\begin{abstract}
The patella is a rare site for osteomyelitis. Because symptoms are mild and variable, diagnosis is often difficult and delayed. We present a case of a 7-year-old boy where diagnosis was only reached by MRI and not by blood sample, ultrasound, bone scintigraphy or X-ray. The patient did well under conservative treatment with antibiotics. We recommend an MRI for early diagnosis as it has a high sensitivity and specificity without any radiation.
\end{abstract}

Keywords: Patellar Osteomyelitis

\section{Introduction}

Patellar osteomyelitis is an uncommon disease, first reported by Thirion in 1829 [1]. Most cases present between the age of 5 and 10 years old. Presentation may be insidious with only mild localized symptoms and is highly variable, which causes diagnosis to be difficult and frequently delayed [2]. Good outcome is to be expected in case of timely diagnosis and treatment [3].

In this report we present the clinical presentation, course and treatment of a patellar osteomyelitis in a 7-year old boy.

\section{Case Report}

\section{History}

An otherwise healthy 7-year old Caucasian boy presented at the emergency department with pain in the left knee, causing an inability to stand or walk. The pain had begun two days earlier. The boy had fallen on his knee, but this was several days before the pain had started. The patient reported to have had impetigo-like lesions around the mouth about 6 weeks prior to the knee pain. This had not been treated but had resolved spontaneously. There was no other history of previous disease.

\section{Clinical examination}

On clinical examination there was no hydrops, only some prepatellar swelling. The knee felt slightly warm. The boy had a tendency to keep his left hip and knee in deep flexion, often even sitting on his knees. Extension of the knee was painful, but there was a full range of motion. Meniscal tests and stability tests were all normal. Palpation of the patella, and even the prepatellar skin was very painful. The clinical examination of the hip was normal on both sides. We also noticed he had scratch marks over both legs and arms.

\section{Technical investigations}

On admission, a blood sample was taken which showed an elevated ESR (Erythrocyte Sedimentation Rate) of $22 \mathrm{~mm} / \mathrm{h}$, a white blood cell count of $11220 / \mu \mathrm{L}$ (no elevation of neutrophils), a CRP of $4.9 \mathrm{mg} / \mathrm{L}$ and a strongly elevated ASLO (Anti-streptolysin O) of $>1000 \mathrm{IU} / \mathrm{ml}$.

An ultrasound of the knee showed soft tissue infiltration anterior to the patella, but no bursitis. Furthermore, a groove in prepatellar fibers was described by the radiologist. It was presumed that a bursitis was developing and treatment with NSAIDs (non-steroidal 
anti-inflammatory drugs) and PRICE (painkillers, rest, ice, compression and elevation) was commenced.

\section{Evolution}

Although treatment with NSAIDs and PRICE was started, the pain level remained much higher than to be expected for a bursitis and thus a bone scintigraphy was performed the next day.

The bone scintigraphy showed a slightly elevated blood flow to the left patella (Figure 1). In the delayed phase there was also an elevated uptake in the entire left patella. Differential diagnosis of trauma (contusion vs. fracture), an infectious or neoplastic process was postulated. As the capitation on the bone scintigraphy was rather low, the most probable diagnosis was a contusion.
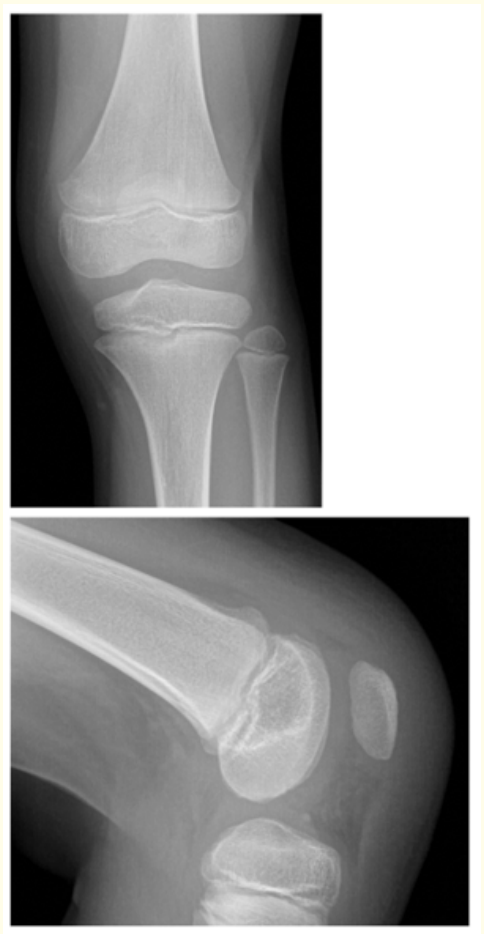

Figure 1: X-ray showing no signs of pathology.

The boy was further monitored, and did not develop any fever or illness, but the pain continued to be disproportionate to the clinical and biochemical findings.

On repeat blood samples the ESR continued to be slightly elevated, with a normal leucocyte count and normal CRP levels. A radiograph of the hip and knee was taken, but showed no signs of disease (Figure 2).

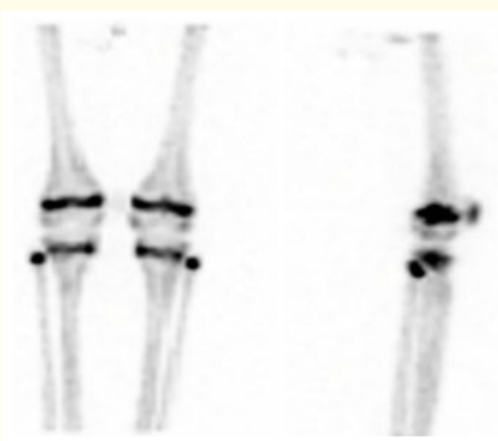

Figure 2: Bone scan showing a slight elevation in uptake at the left patella.

Because of the discrepancy an MRI was performed. The MRI showed a large amount of patellar bone marrow edema with a small fistula connecting the center of the patella to the prepatellar edematous soft tissue without a clear collection (Figure 3). The diagnosis of patellar osteomyelitis was made.

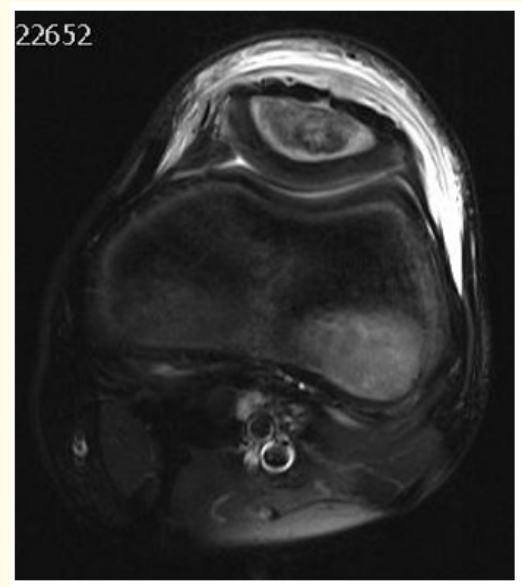

Figure 3: MRI showing bone edema in the patella with a small fistula through the cortex and periosteum.

Thus, we decided to surgically trepan ate the patella under general anesthesia, also taking swabs (bursa, periosteum and intra-osseous) and a bone biopsy for microbiologic and anatomopathologi- 
cal investigation. After surgery, flucloxacillin IV was empirically administered. All specimens proved to be positive for S. aureus, sensitive to flucloxacillin. We placed an open long leg cast for antalgic reasons.

Postoperatively the pain was alleviated even further. A couple of days after surgery, extension of the knee was no longer painful. Two days after surgery the boy developed a fever up to $38.4^{\circ} \mathrm{C}$, which lasted for three days. Concurrently CRP rose until $52.5 \mathrm{mg} /$ dL, ESR till $49 \mathrm{~mm} / \mathrm{h}$ and the white blood cell count until 14010/ $\mu \mathrm{l}$. CRP and white blood cell count both normalized by the $11^{\text {th }}$ day post- surgery, ESR only 20 days post-surgery. IV antibiotics were administered for 10 days, followed by oral therapy for 23 more days.

\section{Discussion}

Patellar osteomyelitis is a very rare condition. At birth the patella is largely cartilaginous $[7,9]$. It starts to ossify between the age of 3 and 6 years old and the ossification is complete at the age of 18 .

Vascularization starts around the age of 4 and reaches a maximum at 12 years of age [3]. The rich vascular network consists of an intra- and extraosseous arterial network. The intraosseous blood supply includes the mi patellar vessels on the anterior surface, the polar vessels from the infrapatellar anastomosis, and an anastomosis posterior to the quadriceps muscle. The extraosseous network includes branches of the superior and inferior geniculate and de anterior tibial recurrent artery. This rich vascularization combined with the absence of a physical plate (associated with sluggish hemodynamics) may explain the rarity of an osteomyelitis in this area $[6,8]$.

The cause is generally considered to be hematogenous, but may be affected by a prior blunt trauma. It is unclear whether the trauma contributed to the infection in this case. No hematoma was seen and there was very little swelling of the prepatellar soft tissues.

The diagnosis of patellar osteomyelitis is often delayed because the symptoms vary and the incidence is very low [2,3,5,7]. In our case the only symptoms were pain and warmth around the patella. There was very little swelling of the prepatellar soft tissues, no redness, no intra-articular effusion. Furthermore, no elevation of CRP or WBC count, only a slight elevation of the ESR, and the patient only developed a fever after the diagnosis was made and surgery had been performed.

Furthermore, a bone scintigraphy and radiograph were insufficient for diagnosis in this case. The bone scintigraphy showed a slight increase in uptake but not enough to suggest an osteomy- elitis.

Performance of the bone scintigraphy on the $3^{\text {rd }}$ day after presentation, early in the course of the disease, is probably the reason of a false negative result in this case. Furthermore, sensitivity and specificity of bone scintigraphy are low.

The radiographs performed half a day earlier than the MRI showed no abnormalities. The MRI scan however did show changes in the patellar bone strongly suggesting osteomyelitis. It seems that MRI has a higher specificity and proved to be more sensitive in this early stage.

If we had opted to perform an MRI scan, the bone scan would not have been necessary, saving the patient from unnecessary radiation.

The most common pathogen causing osteomyelitis is S. Aureus [8], as was found in this case. The gold standard for treatment is surgical debridement and curettage followed by antibiotic therapy although it is suggested that in uncomplicated cases antibiotic treatment alone may be sufficient [3-5]. In our case we performed surgery to obtain biopsies to ensure a correct diagnosis as well as to relieve pain. No consensus exists regarding the use of local antibiotics, in the form of foam or beads [4]. We did not use any of these in our case. There is also no consensus regarding duration of antibiotic treatment [5]. We treated intravenously for 10 days, then switched to oral treatment. This was based on clinical and laboratory findings. Postoperatively, some authors suggest a long leg cast for a period of 6 weeks combined with partial weight bearing. We immobilized with an open long leg cast for pain relief, but encouraged our patient to mobilize and bear weight as possible. Three weeks after surgery the boy was ambulant, had no pain/tenderness or swelling of/around the patella, only a slight warmth was left. Based on the clinical evolution oral antibiotic treatment was continued for 23 days. The warmth over the patella then subsided. The total duration of antibiotic treatment in this patient was 33 days.

\section{Conclusion}

Patellar osteomyelitis is a rare condition which is often difficult to diagnose due to the variable and non-specific symptoms and low incidence. Therefore, it is important to have a high index of suspicion when patellar symptoms persist despite treatment for inflammation, without fever, without striking laboratory-findings, a nonconclusive bone scintigraphy and a normal X-ray. We recommend an MRI for correct and earlier diagnosis as it has a higher sensitivity and specificity and no radiation.

\section{Acknowledgements}

There are no conflicts of interest. 


\section{Bibliography}

1. Thirion M. "Successful extirpation of the right patella for caries". Lancet 2 (1829): 399.

2. Roy DR. "Osteomyelitis of the patella". Clinical Orthopaedics and Related Research 389 (2001): 30-34.

3. Gil-Albarova J., et al. Journal of Pediatric Orthopaedics B 21 (2012): 411-414.

4. Sperl M., et al. "Osteomyelitis of the Patella in a 10-Year-Old Girl: A Case Report and Review of Literature". Case Reports in Orthopedics (2017): 5.

5. De Gheldere A. "Haematogenous osteomyelitis of the patella in a child". Acta Orthopaedica Belgica 75 (2009): 554-556.

6. Shim S and Leung G. "Blood supply of the knee joint: A microangiographic study in children and adults". Clinical Orthopaedics 208 (1986): 119-125.

7. Choi HR. "Patellar osteomyelitis presenting as prepatellar bursitis". The Knee 14 (2007): 333-335.

8. Evans DK. "Osteomyelitis of the patella". Journal of Bone and Joint Surgery 44B (1962): 319-323.

9. Vaninbroukx J., et al. "Haematogenous Osteomyelitis of the Patella”. Acta Orthopaedica Scandinavica 47.5 (1976): 566-569.

Volume 2 Issue 12 December 2019

(C) All rights are reserved by Eva DE Wachter., et al. 